\title{
Interband Bloch oscillation mechanism for high-harmonic generation in semiconductor crystals
}

\author{
C. R. McDonald, ${ }^{1, *}$ G. Vampa, ${ }^{1, \dagger}$ P. B. Corkum, ${ }^{1,2}$ and T. Brabec ${ }^{1}$ \\ ${ }^{1}$ Department of Physics, University of Ottawa, Ottawa, ON K1N 6N5, Canada \\ ${ }^{2}$ National Research Council of Canada, Ottawa, Ontario, Canada K1A OR6
}

(Received 20 May 2015; published 23 September 2015)

\begin{abstract}
High harmonic generation in semiconductors is analyzed for high mid-infrared laser intensities for which the electron-hole pair is driven beyond the first Brillouin zone and exhibits Bloch oscillations. We find that even a two-band analysis exhibits second and higher plateaus. Whereas the first plateau is shown to be consistent with high harmonic generation through electron-hole recollision, the higher plateaus arise from dynamic Bloch oscillations; however, the driving process is interband in nature, in contrast to the generally accepted intraband Bloch oscillation mechanism. Energy conservation is fulfilled, as harmonics beyond the first plateau come from a cascaded nonlinearity.
\end{abstract}

DOI: 10.1103/PhysRevA.92.033845

PACS number(s): 42.65.Ky, 42.50.Hz, 72.20.Ht, 78.47.-p

\section{INTRODUCTION}

The process of high-harmonic generation (HHG) has been studied extensively in atomic and molecular systems [1,2] over the past several decades. Recently, however, interest has grown towards studying HHG in condensed matter systems. Experiments generating high-order harmonics in bulk semiconductor crystals for wavelengths in the mid-infrared (mid-ir) [3,4] and $\mathrm{THz}[5,6]$ regimes have been performed, establishing a foundation on which attosecond electron dynamics in solids can be studied.

HHG in solids can result from two distinct contributionsan interband and an intraband current [7,8]. Theoretical analysis [9,10] and recent experiments [11] revealed that the interband current created by electron hole recollision is the dominant mechanism for mid-ir driver pulses. Its similarity to $\mathrm{HHG}$ in atomic gases allows one to adapt attosecond technology from gases to solids, with potential applications such as, bandgap tomography, solid-state $\mathrm{PHz}$ oscilloscope, and in situ measurement of electric signals in semiconductor devices [11]. By contrast, for longer wavelengths towards the $\mathrm{THz}$ regime, intraband $\mathrm{HHG}$, as a result of the nonlinear band velocity $[6,12-15]$, was found to be dominant $[6,10]$.

Mid-ir HHG experiments [3] have been confined to moderate intensities by the onset of material damage. However, the material damage threshold can be shifted to higher intensities by using shorter pulse durations and/or by going to different materials. This makes the study of the higher intensity regime meaningful and interesting from an application perspective. Higher laser intensities can potentially translate into shorter harmonic wavelengths and shorter pulse durations, both being of interest for attosecond spectroscopy in solids.

At higher intensities, when electrons are driven beyond the first Brillouin zone boundary, two additional processes take place. First, the bandgap between first and second conduction band is usually smallest at the Brillouin zone edge, so that transitions to higher bands may become important [16]. Second, once a conduction electron has crossed the edge of the Brillouin zone, it will begin to move in the opposite

\footnotetext{
*cmcdo059@uottawa.ca

†gvamp015@uottawa.ca
}

direction. This results in the intraband phenomenon known as Bloch oscillation (BO) where the electron moves periodically in space by repeatedly crossing the Brillouin zone boundaries [17].

In interband HHG through recollision in a two-band system, energy conservation limits the harmonic plateau region to the maximum bandgap energy [10]. Recently, in numerical studies a second plateau with harmonics beyond the maximum bandgap [18] was found. Both, higher bands and intraband BOs, were identified as candidates for generating the second plateau.

The main finding of our investigation is a $\mathrm{BO}$ mechanism driving $\mathrm{HHG}$ that is interband in nature, relying on the polarization buildup between valence and conduction band. It manifests as a second plateau, but is fundamentally different from conventional intraband $\mathrm{BO}$ referred to above. For our investigation we use three-dimensional (3D), two- and threeband calculations of $\mathrm{ZnO}$. We have chosen to use $\mathrm{ZnO}$ because it is the only material for which mid-ir HHG experiments have been performed $[3,11]$. The interband $\mathrm{BO}$ mechanism is a new mechanism that has yet to be examined experimentally.

Interband BO-HHG appears for two bands; the role of higher bands is investigated by adding a second conduction band. In $\mathrm{ZnO}$, the second is close to the first conduction band and therefore is potentially important; higher bands are neglected. The presence of the third band affects the harmonic spectrum only weakly, although population in the first and second conduction band are found to be comparable; the electron-hole recollision mechanism remains the dominant source for HHG in the fundamental plateau.

In a more general analysis of 3D model systems, the efficiency of BO-HHG is studied with regard to the parameters of the third band. The efficiency increases substantially with shrinking bandgap between the two conduction bands. Still, interband BOs remain the dominant driving mechanism. As a result, interband $\mathrm{BO}-\mathrm{HHG}$ presents a potential pathway towards extending HHG to shorter wavelengths in selected solids, where closely spaced higher conduction bands enhance its efficiency.

Finally, the mechanism driving HHG from interband BOs is revealed by a saddle point analysis for a two-band model. After the electron is promoted to the conduction band by tunnel ionization, the laser field drives the electron periodically 
through the first Brillouin zone which leads to a fast oscillation in the bandgap energy; the integral over the bandgap energy, the classical action $S$, determines the phase of interband polarization. As integrals over fast oscillations yield zero, HHG through interband BO can happen only around the saddle points in $S$ which occur at each nodal point of the laser field; there BOs are suppressed and the bandgap energy remains approximately constant resulting in a sinusoidal oscillation of the interband polarization and therewith emission of a harmonic photon. Bangap and photon energy are determined by the electron-hole crystal momentum at the field nodal points. Each of these saddle points can create a harmonic spectrum with highest photon energy equal to the maximum bandgap energy, as is required by energy conservation in a two-band model. As the consecutive saddle points can act as a cascaded nonlinearity, the harmonic spectrum can extend over multiples of the maximum bandgap energy.

\section{DERIVATION OF THE DENSITY MATRIX EQUATIONS}

Our analysis is based on a 3D, three-band model of $\mathrm{ZnO}$ (wurtzite structure). Here we will derive the density matrix equations for a solid with an arbitrary number of bands. We will then use these general equations to define our three-band model. We begin with the time-dependent Schrödinger equation (TDSE) in the length gauge where the time-dependent Hamiltonian in atomic units is written as $H(t)=H_{0}-\mathbf{x} \cdot \mathbf{F}(t)$, where $H_{0}=T+U$ is the unperturbed Hamiltonian with $T=(1 / 2) \nabla^{2}$ the kinetic energy and $U(\mathbf{x})$ the periodic potential of the lattice. The field free Hamiltonian $H_{0}$ has Bloch eigenstates $\Phi_{\mathrm{m}, \mathbf{k}}(\mathbf{x})=u_{m, \mathbf{k}}(\mathbf{x}) \exp (i \mathbf{k} \cdot \mathbf{x})$ with energies $E_{m, \mathbf{k}}=E_{m}(\mathbf{k})$ in band $m$ with crystal momentum $\mathbf{k}$; $u_{\mathrm{m}, \mathbf{k}}$ is the periodic part of the Bloch function.

In the presence of the laser field the wave function becomes time dependent. In the length gauge it is represented as

$$
\Psi(\mathbf{x}, t)=\sum_{m} \int_{\mathrm{BZ}} a_{m}(\mathbf{k}, t) \Phi_{m, \mathbf{k}}(\mathbf{x}) d^{3} \mathbf{k},
$$

where $a_{m}(\mathbf{k}, t)$ are the probability amplitudes and integration is over the full Brillouin zone (BZ).

The derivation of the equations of motion for the probability amplitudes proceeds in the same manner as the supplementary material of Ref. [9]. Equation (1) is substituted into the TDSE and the Bloch eigenstates are integrated out yielding,

$$
\dot{a}_{m}=\left(-i E_{m}(\mathbf{k})+\mathbf{F}(t) \nabla_{\mathbf{k}}\right) a_{\mathrm{m}}+i \mathbf{F}(t) \sum_{m^{\prime} \neq m} \mathbf{d}_{\mathrm{mm}^{\prime}}(\mathbf{k}) a_{\mathrm{m}^{\prime}},
$$

where

$$
\mathbf{d}_{m m^{\prime}}(\mathbf{k})=i \int d^{3} \mathbf{x} u^{*}{ }_{m, \mathbf{k}}(\mathbf{x}) \nabla_{\mathbf{k}} u_{m^{\prime}, \mathbf{k}}(\mathbf{x})
$$

is the transition dipole.

Following the Keldysh approach [19], we use the transformation $a_{m}=b_{m} \exp \left(-i \int_{-\infty}^{t} E_{m} d t^{\prime}\right)$ together with $\mathbf{K}=$ $\mathbf{k}-\mathbf{A}(t)$ in Eq. (2) where $\mathbf{A}(t)$ is the vector potential defined by $\mathbf{F}=-d \mathbf{A} / d t$. As a result we arrive at

$$
\dot{b}_{m}(\mathbf{K}, t)=i \sum_{m \neq m^{\prime}} \Omega_{m m^{\prime}}(\mathbf{K}, t) b_{m^{\prime}}(\mathbf{K}, t) e^{i S_{m m^{\prime}}(\mathbf{K}, t)},
$$

where $\Omega_{m m^{\prime}}=\mathbf{F}(t) d_{m m^{\prime}}[\mathbf{K}+\mathbf{A}(t)]$ and

$$
S_{m m^{\prime}}(\mathbf{K}, t)=\int_{-\infty}^{t} \varepsilon_{m m^{\prime}}\left[\mathbf{K}+\mathbf{A}\left(t^{\prime}\right)\right] d t^{\prime}
$$

is the classical action with $\varepsilon_{m m^{\prime}}=E_{m}-E_{m^{\prime}}$ being the bandgap between bands $m$ and $m^{\prime}$.

To connect the Eq. (4) to the density matrix equations we define $n_{m}=\left|b_{m}\right|^{2}$ and $\pi_{m m^{\prime}}=b_{m}^{*} b_{m^{\prime}}$ for $m \neq m^{\prime}$. Putting these definitions into Eq. (2) yields,

$$
\begin{aligned}
\dot{n}_{m}= & i \sum_{m^{\prime} \neq m} \Omega_{m m^{\prime}} \pi_{m m^{\prime}} e^{i S_{m m^{\prime}}}+\text { c.c. } \\
\dot{\pi}_{m m^{\prime}}= & -\frac{\pi_{m m^{\prime}}}{T_{2}}+i \Omega_{m m^{\prime}}^{*}\left(n_{m}-n_{m^{\prime}}\right) e^{-i S_{m m^{\prime}}} \\
& +i \sum_{m^{\prime \prime} \notin\left\{m, m^{\prime}\right\}}\left(\Omega_{m^{\prime} m^{\prime \prime}} \pi_{m m^{\prime \prime}} e^{i S_{m^{\prime} m^{\prime \prime}}}-\Omega_{m m^{\prime \prime}}^{*} \pi_{m^{\prime} m^{\prime \prime}}^{*} e^{-i S_{m m^{\prime \prime}}}\right) .
\end{aligned}
$$

In Eq. (6) we have dropped the input $(\mathbf{K}, t)$ for simplicity. The first term in Eq. (6b) is a phenomenological term that takes into account the dephasing time $T_{2}$. This could be derived by accounting for coupling to a phonon bath and impurities and for electron-electron scattering. The functions $\pi_{m m^{\prime}}(\mathbf{K}, t)$ are connected to the polarization by $\mathbf{p}_{m m^{\prime}}(\mathbf{K}, t)=$ $\mathbf{d}_{m m^{\prime}}(\mathbf{K}, t) \pi_{m m^{\prime}}(\mathbf{K}, t) \exp \left(i S_{m m^{\prime}}(\mathbf{K}, t)\right)+$ c.c. Equation (6) is subject to the constraint $\sum_{m} n_{m}=1$.

The above set of equations is general for any number of bands in a solids. For the three-band model we will use a single valence band and two conduction bands. There is a dipole transition moment between the valvence and first conduction band and between the first and second conduction bands. However, the dipole transition moment between the valence and second conduction band is set to zero. From Eq. (6) we will have six equations to solve in our three-band model. The workload to solve this system will be approximately double that required for the two-band model.

\section{NUMERICAL CALCULATIONS}

\section{A. Description of $\mathrm{ZnO}$ model}

For our calculations we use a 3D, three-band model of $\mathrm{ZnO}$ (wurtzite structure). However, most of our analysis is confined to the lowest two bands; three-band results are discussed at the end of the paper. The reciprocal lattice is oriented so that $\hat{\mathbf{x}}$ is along $\Gamma-M, \hat{\mathbf{y}}$ along $\Gamma-K$, and $\hat{\mathbf{z}}$ along $\Gamma-A$ (optical axis); the lattice constants are $\left(a_{x}, a_{y}, a_{z}\right)=$ $(5.32,6.14,9.83)$ a.u. In the two-band model, the bandgap is determined by $\varepsilon_{g}=E_{c}(\mathbf{k})-E_{v}(\mathbf{k})=E_{g}+\Delta E_{g}(\mathbf{k})$ where $E_{c}$ is the conduction band (electrons) and $E_{v}$ is the valence band (holes). The bands were determined by the nonlocal empirical pseudopotential method (NL-EPM) [20]; the complete $3 \mathrm{D}$ band in the first Brillouin zone is approximated as the sum over the three one-dimensional bands. Since the wave vector $(\mathbf{k} \| \hat{\mathbf{z}})$ is much smaller in magnitude than the reciprocal lattice vectors, the dipole approximation is used.

For our three-band model we use a single valence band $(V)$ and two conduction bands obtained from Ref. [20]. Figure 1 shows the structure along $\Gamma-M$. The lowest conduction band $\left(C_{1}\right)$ couples to both the valence and higher conduction band 


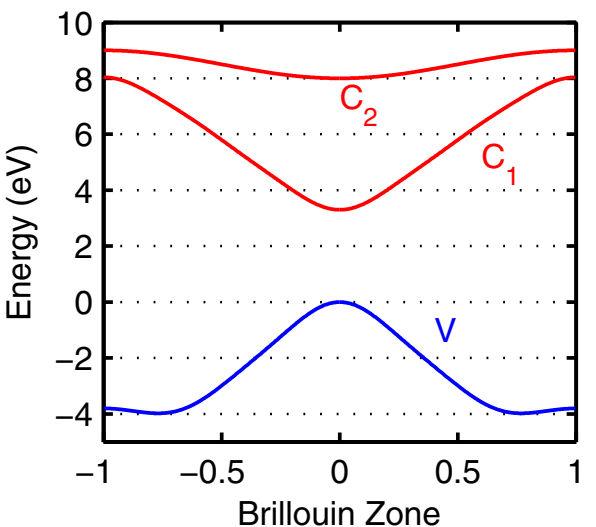

FIG. 1. (Color online) Band structure along $\Gamma-M$ for threeband model with a single valence band (blue) and two conduction bands (red). In the two-band model only the valence and lowest conduction band are used.

$\left(C_{2}\right)$; however, there is no coupling between $V$ and $C_{2}$. At the $\Gamma$ point the bandgap between $V$ and $C_{1}$ is $3.3 \mathrm{eV}$. By contrast the bandgap at the $\Gamma$ point between $V$ and $C_{2}$ is $8 \mathrm{eV}$. This makes it unlikely that population will be transferred directly from $V$ to $C_{2}$ for the field parameters that we use.

At the edge of the Brillouin zone the bandgap between $C_{1}$ and $C_{2}$ is $\approx 1 \mathrm{eV}$. For field strengths that are strong enough to drive an electron near, or beyond, the $\mathrm{BZ}$ edge it becomes possible to transfer population between the two conduction bands. The conduction band above $C_{2}$ is far enough away that it should not have a significant effect.

Interaction with the intense laser field is calculated using the density matrix equations derived in the previous section. For the $\mathbf{k}$ dependence of the dipole we use the same model as presented in the supplementary material of Ref. [9]. Here each dipole element is calculated by

$$
d_{j}(\mathbf{k})=\sqrt{\frac{E_{p, j}}{2 \varepsilon_{g}^{2}(\mathbf{k})}},
$$

were $j=x, y, z, \varepsilon_{g}$ is the bandgap, and $E_{p, j}$ are the Kane parameters [21-24]. For our calculations we use $E_{p, x}=$ $E_{p, y}=0.302$ a.u. and $E_{p, z}=0.375$ a.u. for both the $C_{1}-V$ and the $C_{1}-C_{2}$ pairs. The crystal is exposed to a laser field $\mathbf{F}(t)=\hat{\mathbf{x}} F_{0} f(t)$ where $f(t)$ consists of a sine carrier with wavelength $\lambda=3.25 \mu \mathrm{m}$ and temporal Gaussian envelope with a full width at half maximum (FWHM) equal to 10 cycles. For momentum-space integration 600 points along $\Gamma-M$ are used for the full Brillouin zone; 200 points are used along the other two directions. The interband contribution to the harmonic spectrum is calculated by taking the Fourier transform of the interband current (see Ref. [9]).

\section{B. Two-band results}

Before solving the full three-band equations we will investigate the two-band system where we only consider bands $V$ and $C_{1}$. Figure 2 shows the harmonic spectra for field strengths $F_{0}=0.007$ a.u. (blue) and $F_{0}=0.01$ a.u. (red) in the crystal. These field strengths correspond to vacuum field intensities of $I_{\mathrm{v}}=3.6 \mathrm{TW} / \mathrm{cm}^{2}\left(F_{\mathrm{v}}=0.51 \mathrm{~V} / \AA\right)$ and

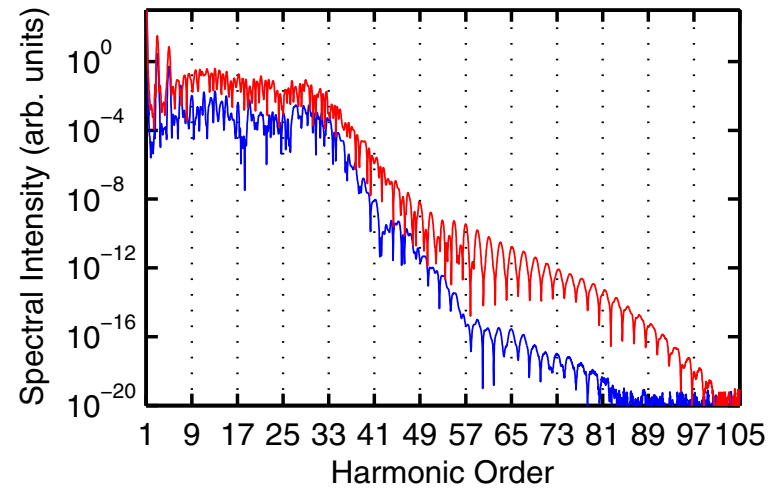

FIG. 2. (Color online) Harmonic spectra for $F_{0}=0.007$ a.u. (blue) and $F_{0}=0.01$ a.u. (red) for $\lambda=3.25 \mu \mathrm{m}$, and for a dephasing time of $T_{2}=5.4 \mathrm{fs}$ (equal to half cycle).

$I_{\mathrm{v}}=7.4 \mathrm{TW} / \mathrm{cm}^{2}\left(F_{\mathrm{v}}=0.73 \mathrm{~V} / \AA\right)$, respectively. By contrast, the highest vacuum intensity used for experimental measurement in Ref. [3] was $5 \mathrm{TW} / \mathrm{cm}^{2}(0.6 \mathrm{~V} / \AA)$. The relation between $F_{0}$ and $F_{\mathrm{v}}$ is $F_{0}=2 F_{\mathrm{v}} /(n+1)$ where the index of refraction for our system is $n \approx 1.9$. A dephasing time of $T_{2}=5.4 \mathrm{fs}$ - equivalent to a laser half cycle-is used in our calculations. At both field strengths electrons will travel beyond the first Brillouin zone and a second plateau emerges followed by another exponential drop; for higher field strengths the second plateau is more pronounced. This "staircase" structure is not observed when the field is too weak for electrons to travel beyond the first Brillouin zone. Finally, the staircase structure appears also in the intraband current, however, is considerably weaker, and therefore not shown here.

Figure 3 shows the results of a windowed Fourier transform of the interband current for $F_{0}=0.01$ a.u. using a 0.34 cycle Blackman window scanned across two optical cycles near the peak of the pulse. This narrow temporal window allows only a single recollision event and thus the resulting spectrum is continuous rather than composed of discrete harmonics. This process is carried out with a spectral filter placed near the first cutoff [Fig. 3(a)] and is repeated with a spectral filter placed near the second cutoff [Fig. 3(b)].

Figure 3(a) shows the time-frequency analysis for the fundamental plateau. The highest photon energy is emitted slightly before a field zero; this structure will repeat itself every half-cycle. The white lines show the results of the classical trajectories calculated from the recollision model similar to Ref. [10]; here there are two branches. The branch on the right corresponds to trajectories that stay within the first Brillouin zone; these trajectories are born after the peak of the field. The branch on the left results from trajectories born before the peak of the field; these can extend into the second Brillouin zone. In contrast to gases-where trajectories born before the peak don't recollide-it is these trajectories that dominate the harmonic spectrum of the first plateau in our solid. While the Bloch oscillation influences the classical trajectory, it is clear that recollision is the main driver of the harmonic spectrum for the first cutoff.

Figure 3(b) shows the results of our time-frequency analysis for the second cutoff. In this case all harmonics are emitted in 


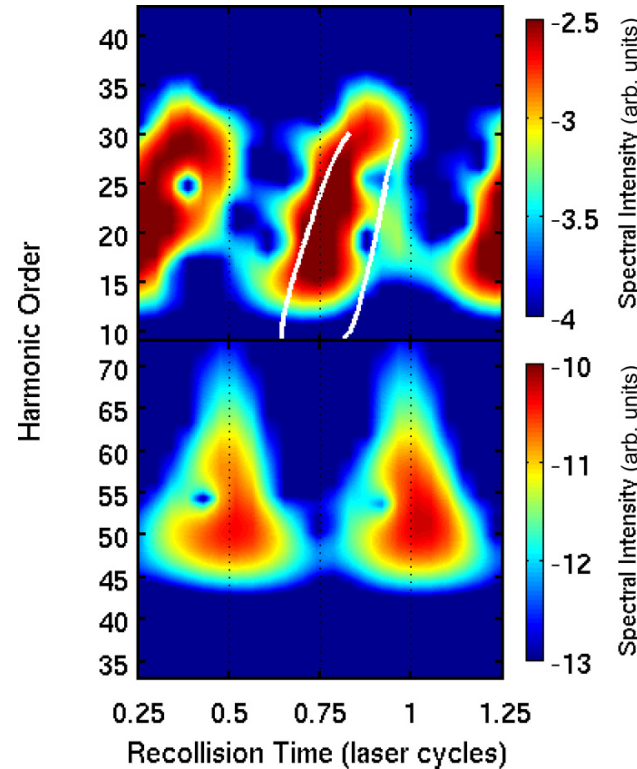

FIG. 3. (Color online) Harmonic order versus time for the timefrequency analysis for the (a) fundamental and (b) second cutoff for field intensity $F_{0}=0.01$ a.u. with $\lambda=3.25 \mu \mathrm{m}$ and $T_{2}=5.4 \mathrm{fs}$. The field peaks occur at quarter-cycle times and the nodes occur at integer and half-integer cycle times. The white lines are the results for the first returns from the semiclassical trajectory analysis. The color scale is logarithmic.

phase near a field zero. The observed difference is indicative of an interband process for emitting harmonic radiation that occurs when electrons traverse beyond the first Brillouin zone that is fundamentally different from the classical trajectory picture. While emission in the first plateau is still timed to the recolliding electron-hole pairs, harmonics in the second plateau are all emitted in phase at the field node.

\section{SADDLE POINT ANALYSIS OF INTERBAND BLOCH OSCILLATIONS}

In order to get a physical picture of the process creating the harmonics beyond the maximum bandgap, we perform a saddle point analysis in the Bloch oscillation limit where the electron traverses the Brillouin zone many times. We begin with the expression for the interband current from Ref. [9],

$$
\begin{aligned}
j_{\mathrm{er}}(\omega)= & \omega \int_{\mathrm{BZ}} d^{3} \mathbf{k} d(\mathbf{k}) \int_{-\infty}^{\infty} d t e^{i \omega t} \int_{-\infty}^{t} d t^{\prime} F\left(t^{\prime}\right) d^{*}\left(\boldsymbol{\kappa}_{t^{\prime}}\right) \\
& \times e^{-i S\left(\mathbf{k}, t^{\prime}, t\right)-\left(t-t^{\prime}\right) / T_{2}}+\text { c.c. }
\end{aligned}
$$

For our physical picture in the Bloch oscillation limit we are interested in the exponential term. Integration over $\mathbf{k}$ and $t^{\prime}$ will yield

$$
j_{\mathrm{er}}(\omega)=\sum_{t_{b}} Q\left(t_{b}\right) \int_{-\infty}^{\infty} d t e^{i \omega t} e^{-i S\left(t_{b}, t\right)}+\text { c.c. }
$$

where $t_{b}$ is the birth time, $Q$ is the pre-exponential factor, $S\left(t_{b}, t\right)=\int_{t_{b}}^{t} \varepsilon_{g}\left[A\left(t^{\prime \prime}\right)-A\left(t_{b}\right)\right] d t^{\prime \prime}$, and $A(t)$ is the vector potential with $d A / d t=-F(t)$. Here we are using the saddle point condition $k=A(t)-A\left(t_{b}\right)$ derived in Ref. [9].

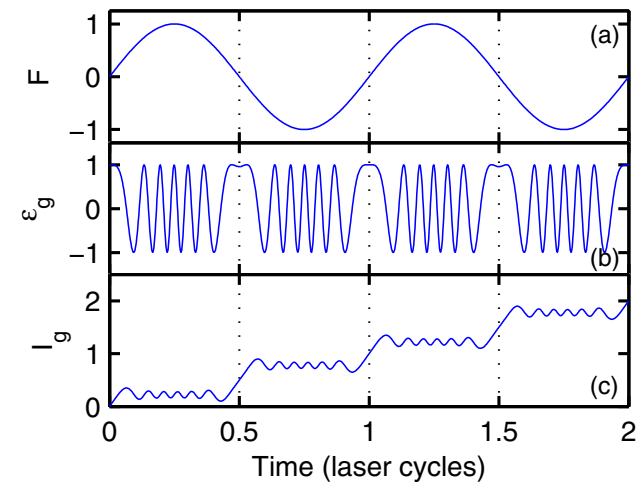

FIG. 4. (Color online) (a) Laser field normalized by peak amplitude. (b) Integrand of $I_{g}$; that is $\Delta^{-1}\left(\varepsilon_{g}-E_{g}\right)$. The saddle points occur at the field nodes. (c) Full integral $I_{g}$. The steps result from the contributions from the individual saddle points. Here we use the fundamental frequency $\omega_{0}=0.014$ a.u., lattice constant $a=$ 5.32 a.u., and $F_{0}=0.04$ a.u. The field strength is made artificially strong for illustrative purposes.

Furthermore, for the purpose of our analysis we have set $T_{2}=\infty$. Moreover, to simplify our expressions we will use the tight binding approximation for the bandgap $\varepsilon_{g}=E_{g}+$ $\Delta\left\{1-\cos \left[a\left(A\left(t^{\prime \prime}\right)-A\left(t_{b}\right)\right)\right]\right\}$ where $a$ is the lattice constant. Looking at a single birth time and ignoring the pre-expontential factor, we can then write the polarization as

$$
\tilde{p}_{t_{b}}(\omega) \propto \int_{-\infty}^{\infty} e^{i \omega t} e^{-i\left(E_{g}+\Delta\right)\left(t-t_{b}\right)} e^{i \Delta I_{g}\left(t_{b}, t\right)} d t
$$

where $I_{g}\left(t_{b}, t\right)=\int_{t_{b}}^{t} \cos \left[a\left(A\left(t^{\prime \prime}\right)-A\left(t_{b}\right)\right)\right] d t^{\prime \prime}$. In Fig. 4 the laser field $F, \varepsilon_{g}$, and $I_{g}$ are plotted as a function of time. Note that $\varepsilon_{g}$ is a rapidly oscillating function so that the integral $I_{g}$ in the exponent has to be calculated with the saddle point method. This is fundamentally different from the usual use of saddle point integration for $\mathrm{HHG}$, which is applied to the integral over the exponent, $\int d t^{\prime} \exp \left(i S\left(t^{\prime}, t\right)\right)[25]$.

Defining the phase term in $I_{g}\left(t_{b}, t\right)$ as $\phi\left(t_{b}, t^{\prime \prime}\right)=a\left(A\left(t^{\prime \prime}\right)-\right.$ $\left.A\left(t_{b}\right)\right)$ at the saddle point $t_{s}$ we get the condition,

$$
\left.\frac{d \phi}{d t^{\prime \prime}}\right|_{t^{\prime \prime}=t_{s}}=-a F\left(t_{s}\right)=0 .
$$

This implies that the saddle points occur at the nodal points of the field (when the vector potential is at a maximum); this can be seen in Fig. 4(b). That is, the saddle points are given by $t_{s}=n \pi / \omega_{0}$, where $\omega_{0}$ is the fundamental frequency of the driving field and $n \in \mathbb{Z}$. Thus, at the saddle point $t_{s}$ we have $\phi\left(t_{b}, t_{s}\right)=a\left(A\left(t_{s}\right)-A\left(t_{b}\right)\right)$ and $\phi^{\prime \prime}\left(t_{b}, t_{s}\right)=-a \dot{F}\left(t_{s}\right)$.

In the neighborhood of a saddle point $t_{s}$ we then have the integral,

$$
I_{g}^{(s)} \approx \int_{t_{b}-t_{s}}^{t-t_{s}} \cos \left[\phi\left(t_{b}, t_{s}\right)+\frac{1}{2} \phi^{\prime \prime}\left(t_{b}, t_{s}\right) \tau^{2}\right] d \tau .
$$

Letting $\phi_{s}=\phi\left(t_{b}, t_{s}\right)$ and $\beta=\sqrt{a \dot{F}\left(t_{s}\right) / 2}$, Eq. (12) can be written as

$$
I_{g}^{(s)} \approx \beta^{-1} \int_{\beta\left(t_{b}-t_{s}\right)}^{\beta\left(t-t_{s}\right)} \cos \left(\phi_{s}-\tau^{2}\right) d \tau .
$$


This can be further simplified using the trigonometric identity $\cos (u-v)=\cos u \cos v+\sin u \sin v$, becoming

$$
\begin{aligned}
I_{g}^{(s)} \approx & \beta^{-1}\left\{\cos \left(\phi_{s}\right) C\left[\beta\left(t_{b}-t_{s}\right), \beta\left(t-t_{s}\right)\right]\right. \\
& \left.+\sin \left(\phi_{s}\right) G\left[\beta\left(t_{b}-t_{s}\right), \beta\left(t-t_{s}\right)\right]\right\} .
\end{aligned}
$$

The functions $C$ and $G$ are given by

$$
\begin{aligned}
& C\left[\beta\left(t_{b}-t_{s}\right), \beta\left(t-t_{s}\right)\right]=\int_{\beta\left(t_{b}-t_{s}\right)}^{\beta\left(t-t_{s}\right)} \cos \left(\tau^{2}\right) d \tau, \\
& G\left[\beta\left(t_{b}-t_{s}\right), \beta\left(t-t_{s}\right)\right]=\int_{\beta\left(t_{b}-t_{s}\right)}^{\beta\left(t-t_{s}\right)} \sin \left(\tau^{2}\right) d \tau,
\end{aligned}
$$

where $C(0, x)$ and $G(0, x)$ are Fresnel integrals. Performing a Taylor expansion of the above expressions yields

$$
\begin{gathered}
C\left[\beta\left(t_{b}-t_{s}\right), \beta\left(t-t_{s}\right)\right]=\tau-\frac{\tau^{5}}{10}+\left.\ldots\right|_{\beta\left(t_{b}-t_{s}\right)} ^{\beta\left(t-t_{s}\right)}, \\
G\left[\beta\left(t_{b}-t_{s}\right), \beta\left(t-t_{s}\right)\right]=\frac{\tau^{3}}{3}-\frac{\tau^{7}}{30}+\left.\ldots\right|_{\beta\left(t_{b}-t_{s}\right)} ^{\beta\left(t-t_{s}\right)} .
\end{gathered}
$$

Only the function $C$ has a linear term; this can be observed in Fig. 4(c). Thus, retaining only the linear term gives

$$
I_{g}^{(s)} \approx \cos \left(\phi_{s}\right)\left(t-t_{b}\right) .
$$

As saddle point integration was performed on the integral in the exponent, the sum over individual saddle points in the exponent is equivalent to a product of exponents, each belonging to an individual saddle point contribution. As a result, the Fourier integral for the polarization becomes

$$
\tilde{p}_{t_{b}}(\omega) \propto \prod_{t_{s}} \int_{-\infty}^{\infty} e^{-i\left(E_{g}+\Delta-\omega\right)\left(t-t_{b}\right)} e^{i \Delta I_{g}^{(s)}} d t .
$$

Inserting Eq. (17) into the above expression and integrating over $t$ results in a delta function yielding the relation,

$$
\omega=E_{g}+\Delta\left\{1-\cos \left(\phi_{s}\right)\right\} .
$$

Maximum ionization occurs at field peaks for which $A\left(t_{b}\right)=0$. Then, $\omega$ becomes maximum when the last term in Eq. (19), $\cos \left[a A\left(t_{s}\right)\right]=-1$. That is fulfilled when the laser is strong enough so that the electron reaches the edge of the Brillouin zone during its excursion, i.e., for $F_{0} / \omega_{0} \geqslant \pi / a$, or for $F_{0} \geqslant F_{b}$ with $F_{b}=\pi \omega_{0} / a$ the Bloch field strength. For our system with $a=5.32$ a.u. and $\omega_{0}=0.014$ a.u. we have $F_{b} \approx 0.008$ a.u. As a result, for $F=F_{b}$ a single saddle point can produce a harmonic with cutoff equal to the fundamental plateau.

This implies that in Eq. (18) we have a cascaded nonlinearity, the Fourier transform of a product of functions each having the possibility to produce a spectral range equal to the fundamental plateau. Put in another way, the electron can only collect the bandgap energy. However, it can collect this energy at each nodal point of the laser field in a way that emission at nodal points act as a cascaded nonlinearity. The cascaded nature of the process allows the generation of harmonics up to multiples of the fundamental cutoff. However, as the efficiency goes with the power of the number of saddle points contributing to the cascaded nonlinearity, there is a rapid drop in efficiency, as can be seen in Fig. 2.

\section{EFFECT OF THE SECOND CONDUCTION BAND}

Finally, we will investigate the effect of including a second conduction band into our model as described in Sec. III A.

Figure 5(a) shows a comparison of the two-band (red) and three-band (green) models for $\mathrm{ZnO}$ for $F_{0}=0.01$ a.u. Here it can be seen that the the addition of the second conduction band has little effect on the overall harmonic spectrum. Furthermore, we have investigated the influence of the shape of the second conduction band on HHG via BOs by altering its width, the bandgap between the two conduction bands at the Brillouin zone, and by flipping the band at the $\Gamma$ point, thus turning it from valley to peak; only the bandgap seems to have a significant effect on the spectrum. Increasing the bandgap between the conduction bands at the Brillouin zone edge results in little change of the three-band spectrum. However, reducing it from $1 \mathrm{eV}(\mathrm{ZnO})$ to $0.75 \mathrm{eV}$ results in a significant enhancement of the second plateau; see the purple line in Fig. 5(a). This suggests that there is the possibility to extend HHG to higher frequencies by using materials with a
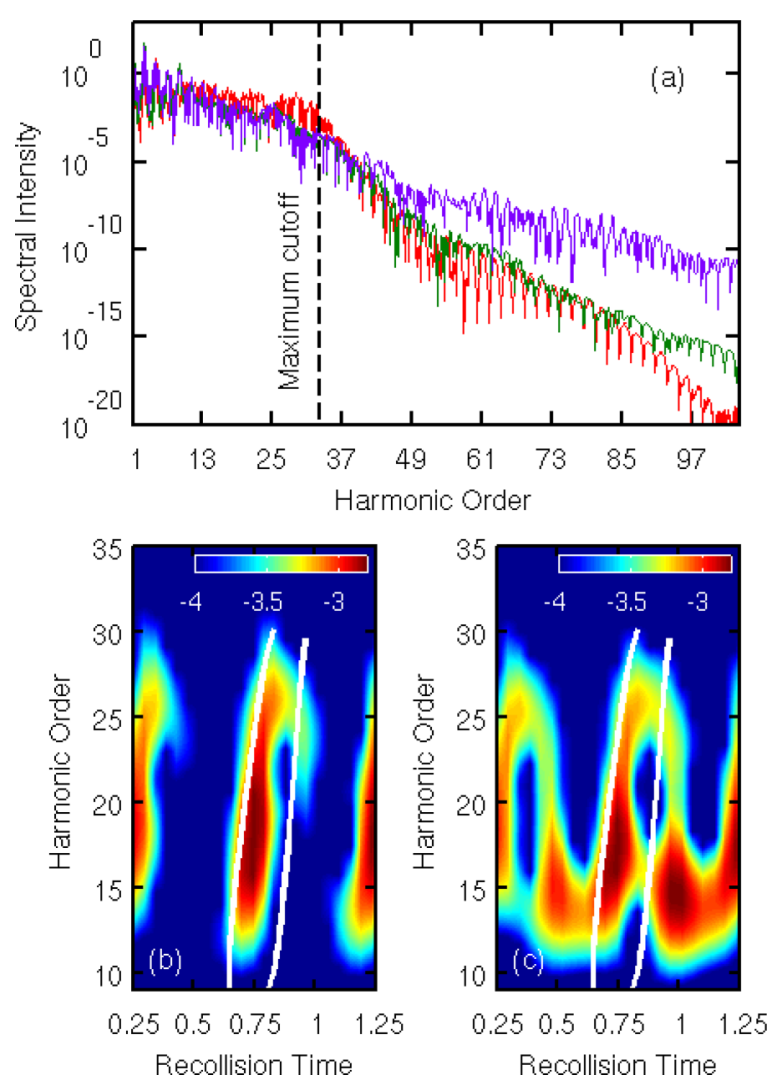

FIG. 5. (Color online) (a) Comparison of the harmonic spectra at $F_{0}=0.01$ a.u. for the two-band (red) and three-band (green) $\mathrm{ZnO}$ models. In addition a harmonic spectrum with a reduced bandgap between the conduction bands at the Brillouin zone edge is shown (purple). (b) Time-frequency analysis of the first cutoff for the threeband model. (c) Time-frequency analysis of the first cutoff for the three-band model with reduced bandgap between conduction bands at the Brillouin zone edge. The white lines are the results for the first two electron-hole recollisions from the semiclassical trajectory analysis for the two-band model. The color scale is logarithmic. 
reduced bandgap at the Brillouin zone edge. The nature of this enhancement is subject to future research.

In Fig. 5(b), the time-frequency analysis centered at the first cutoff of the (green) three-band spectrum in Fig. 5(a) is shown; Recollision is still the dominant mechanism for harmonics below the maximum bandgap. The time frequency analysis of the (purple) spectrum in Fig. 5(a) for the reduced bandgap shows that recollision still plays a dominant role below the maximum bandgap. However, the BO contribution to the first plateau at field zero times 0.5 and 1 has also become visible; see Fig. 5(c). This demonstrates that the $\mathrm{BO}$ mechanism remains intact in multiband systems and is responsible for the enhanced second plateau.

\section{CONCLUSION}

We have investigated theoretically HHG in semiconductors for mid-ir laser fields strong enough to drive the electron-hole pair beyond the first Brillioun zone. So far the generation of radiation via Bloch oscillations has been viewed as an intraband mechanism, where radiation is created by the nonlinear motion of electrons and holes in their respective bands. Here we have identified HHG via interband Bloch oscillations, which is different in nature as it relies on the buildup of polarization between electrons and holes. Building on our previous work, we have shown that, even in the Bloch oscillation limit, recollison remains the dominant mechanism for HHG up to the first cutoff.

The harmonics generated by interband Bloch oscillations appear to be too weak to be observed experimentally by current $\mathrm{ZnO}$ experiments. However, in combination with higher bands, interband Bloch oscillations appear to be a promising mechanism to push $\mathrm{HHG}$ in solids towards shorter wavelengths. In this spectral region, materials typically become more transparent than in the ultraviolet, effectively enhancing interband Bloch oscillations. For example, in silicon the absorption length increases from a minimum of $7 \mathrm{~nm}$ at $\sim 5.6 \mathrm{eV}$ to $66 \mathrm{~nm}$ at $\sim 31 \mathrm{eV}$ [26] - an increase of 4 orders of magnitude in transmission at such distance from the surface. It is conceivable to engineer materials that are capable of exploiting interband Bloch oscillations to increase the harmonic efficiency of harmonics beyond the first cutoff.

\section{ACKNOWLEDGMENTS}

All authors acknowledge support from The National Sciences and Engineering Research Council of Canada (NSERC). P.B.C. and G.V. also acknowledge support from the US Air Force Office of Scientific Research (AFOSR) and National Research Council Canada.
[1] P. B. Corkum, Phys. Rev. Lett. 71, 1994 (1993).

[2] P. B. Corkum and F. Krausz, Nat. Phys. 3, 381 (2007).

[3] S. Ghimire et al., Nature Phys. 7, 138 (2011).

[4] S. Ghimire, A. D. DiChiara, E. Sistrunk, U. B. Szafruga, P. Agostini, L. F. DiMauro, and D. A. Reis, Phys. Rev. Lett. 107, 167407 (2011).

[5] B. Zaks, R. B. Liu, and M. S. Sherwin, Nature (London) 483, 580 (2012).

[6] O. Schubert et al., Nat. Photonics 8, 119 (2014).

[7] H. Haug and S. W. Koch, Quantum Theory of the Optical and Electronic Properties of Semiconductors (World Scientific, Singapore, 2004).

[8] T. Higuchi, M. I. Stockman, and P. Hommelhoff, Phys. Rev. Lett. 113, 213901 (2014).

[9] G. Vampa, C. R. McDonald, G. Orlando, D. D. Klug, P. B. Corkum, and T. Brabec, Phys. Rev. Lett. 113, 073901 (2014).

[10] G. Vampa, C. R. McDonald, G. Orlando, P. B. Corkum, and T. Brabec, Phys. Rev. B 91, 064302 (2015).

[11] G. Vampa et al., Nature (London) 522, 462 (2015).

[12] A. F. Kemper, B. Moritz, J. K. Freericks, and T. P. Deveraux, New J. Phys. 15, 023003 (2013).

[13] P. G. Hawkins and M. Yu. Ivanov, Phys. Rev. A 87, 063842 (2013).

[14] K. A. Pronin, A. D. Bandrauk, and A. A. Ovchinnikov, Phys. Rev. B 50, 3473 (1994).
[15] S. Ghimire, A. D. DiChiara, E. Sistrunk, G. Ndabashimiye, U. B. Szafruga, A. Mohammad, P. Agostini, L. F. DiMauro, and D. A. Reis, Phys. Rev. A 85, 043836 (2012).

[16] P. G. Hawkins, M. Y. Ivanov, and V. S. Yakovlev, Phys. Rev. A 91, 013405 (2015).

[17] P. Földi, M. G. Benedict, and V. S. Yakovlev, New J. Phys. 15, 063019 (2013).

[18] M. Wu, S. Ghimire, D. A. Reis, K. J. Schafer, and M. B. Gaarde, Phys. Rev. A 91, 043839 (2015).

[19] L. V. Keldysh, J. Exp. Theor. Phys. 47, 1945 (1964) [Sov. Phys. JETP 20, 1307 (1965)].

[20] M. Goano, F. Bertazzi, M. Penna, and E. Bellotti, J. App. Phys. 102, 083709 (2007).

[21] I. Vurgaftman et al., J. Appl. Phys. 89, 5815 (2001).

[22] U. Ozgur et al., J. Appl. Phys. 98, 041301 (2005).

[23] A. Schleife, F. Fuchs, C. Rödl, J. Furthmüller, and F. Bechstedt, Phys. Status Solidi B 246, 2150 (2009).

[24] Q. Yan, P. Rinke, M. Winkelnkemper, A. Qteish, D. Bimberg, M. Scheffler, and C. G. Van de Walle, Appl. Phys. Lett. 101, 152105 (2012).

[25] M. Lewenstein, P. Balcou, M. Y. Ivanov, A. L'Huillier, and P. B. Corkum, Phys. Rev. A 49, 2117 (1994).

[26] E. D. Palik, Handbook of Optical Constants of Solids (Academic Press, San Diego, 1998), Vol. 1, pp. 571-586. 